\title{
Perspective
}

Actualité en histoire de l'art

\section{Revisiter les « arts du monde » : histoires critiques et défis contemporains}

Revisiting "World Art": Critical Histories and Contemporary Challenges

„Weltkünste“ neu denken: kritische Geschichten und zeitgenössische

Herausforderungen

Rivisitare le "arti del mondo": storie critiche e sfide contemporanee

Revisitar las "artes del mundo": historias críticas y retos contemporáneos

\section{Felicity Bodenstein et Eva-Maria Troelenberg}

Traducteur : Françoise Jaouën

\section{OpenEdition}

Journals

Édition électronique

URL : https://journals.openedition.org/perspective/7332

DOI : 10.4000/perspective.7332

ISSN : 2269-7721

Éditeur

Institut national d'histoire de l'art

Édition imprimée

Date de publication : 30 juin 2017

Pagination : 169-176

ISBN : 9782917902387

ISSN : 1777-7852

\section{Référence électronique}

Felicity Bodenstein et Eva-Maria Troelenberg, « Revisiter les « arts du monde » : histoires critiques et défis contemporains », Perspective [En ligne], 1 | 2017, mis en ligne le 31 décembre 2017, consulté le 16 janvier 2023. URL : http://journals.openedition.org/perspective/7332 ; DOI : https://doi.org/

10.4000/perspective.7332 


\section{Revisiter les « arts du monde " : histoires critiques et défis contemporains}

\section{Felicity Bodenstein et Eva-Maria Troelenberg}

- Didi-HUBERMAN, 2013 : Georges Didi-Huberman, L'album de l'art à l'époque du "Musée imaginaire", Paris, Hazan/musée du Louvre, 2013, 206 p., 30 ill. coul., 51 ill. n\&b.

- GRASSKAMP, 2014 : Walter Grasskamp, André Malraux und das imaginäre Museum: die Weltkunst im Salon, Munich, C. H. Beck, 2014, 232 p., 65 ill.

- Leeb, 2015 : Susanne Leeb, Die Kunst der Anderen: "Weltkunst" und die anthropologische Konfiguration der Moderne, Berlin, b-books Verlag, 2015, 320 p., 26 ill. n\&b.

- SINGH, 2015 : Kavita Singh, Museums, Heritage, Culture: Into the Conflict Zone, Amsterdam, Reinwardt Academie, Amsterdamse Hogeschool voor de kunsten, 2015, 81 p., 24 ill. coul.

- THOMAs, 2016 : Nicholas Thomas, The Return of Curiosity: What Museums are Good For in the 21st Century, London, Reaktion Books, 2016, 144 p., 35 ill.

Cette liste rassemble quelques ouvrages récents portant sur l'histoire de la catégorie conceptuelle des " arts du monde " et sur les pratiques contemporaines du patrimoine "global ". Ensemble, ils composent un cadre théorique permettant d'analyser un ensemble de pratiques visuelles et de questions sur la circulation des images et des objets (et leur interprétation) d'une culture à l'autre, à travers le temps et au-delà des frontières. La légitimité de collectionner, d'interpréter et d'exposer des objets ou des images issus d'autres cultures fait aujourd'hui de plus en plus débat, du point de vue du droit comme sur le terrain historiographique et conceptuel ${ }^{1}$. Depuis son avènement, "l'histoire de l'art globale " est considérée comme une rupture majeure à l'échelle de l'histoire de la discipline, car elle apporte un nouvel éclairage, non seulement sur l'état de la discipline aujourd'hui, mais également sur sa genèse et la manière dont elle conçoit son rôle, notamment depuis le début du $\mathrm{XX}^{\mathrm{e}}$ siècle, période à laquelle s'est imposée la notion d' " arts du monde ${ }^{2}$ ".

L'une des grandes questions qui resurgit dans de nombreux débats sur l'" art global " porte sur la délicate notion d'universalisme ${ }^{3}$. Peut-on dresser une carte englobante de l'histoire de l'art, et inventer une méthode générale dérivée de la discipline traditionnelle qui permettrait de traiter des nombreuses formes d'art présentes et passées, quelle que soit leur origine géographique? Comment parler de la différence sans universaliser les couples binaires ou les hiérarchies fondées sur le concept d'évolution linéaire ${ }^{4}$ ? De récentes études se sont intéressées à l'ensemble de ces questions en utilisant des approches variées, à partir de divers projets et cas d'étude. Un axe fructueux d'investigation consiste à s'interroger sur la manière dont les " biographies " interculturelles des objets ou des œuvres d'art sont affectées par les conditions épistémiques de leur perception définies par les catégories de l'histoire de l'art, ses médias et ses institutions. Les ouvrages évoqués ici proposent diverses approches de la question générale de "l'histoire de l'art globale ", ouvrant ainsi la voie à un repérage du champ transculturel, de l'intérieur, mais aussi en lien avec l'histoire de la discipline.

La réflexion est menée à partir du concept utopiste de "musée imaginaire " élaboré par André Malraux au milieu du $\mathrm{XX}^{\mathrm{e}}$ siècle, et sur lequel se sont récemment penchés les historiens de l'art Georges Didi-Huberman et Walter Grasskamp dans deux études publiées presque simultanément, en 2013 et en $2014^{5}$. Il faut corréler ce regain d'intérêt des historiens de l'art pour le travail de Malraux ${ }^{6}$ à un besoin de procéder au réexamen critique du principe universaliste qu'il incarne. Il s'inscrit par ailleurs dans une investigation plus ample touchant au rôle joué par différents médias dans l'évolution d'une histoire de l'art comparative et transnationale. Didi-Huberman retrace ainsi la généalogie du projet de Malraux : depuis une approche radicale de l'art, il a progressivement évolué vers une forme de "bien-pensance " qui néglige les asymétries politiques et les relations hiérarchiques inhérentes à la production de la catégorie des arts du monde. Grasskamp, de son côté, fournit une passionnante analyse de la genèse du " musée sans murs " en lien avec l'histoire des médias, en étudiant l'impact de la reproduction photographique et de la circulation des images, évoquant le trope du musée dans sa dimension visuelle concrète, tout en l'inscrivant dans l'histoire des idées?

Les ouvrages de Kavita Singh (2015) et de Nicholas Thomas (2016) permettent de rattacher le musée imaginaire/conceptuel à son incarnation concrète, et de s'interroger sur les conséquences de la collection et de l'interprétation d'objets et 
d'œuvres dans un monde postcolonial ou postmoderne, essentiellement incompatible avec des concepts totalisants. Chacun à leur manière, les deux auteurs confrontent la notion d' " arts du monde " et le discours esthétique en général à d'autres valeurs et concepts d'art et de patrimoine culturel. Les trois cas d'étude (ou "méprises muséales ${ }^{8}$ ") choisis par Singh en Asie du Sud démontrent que si la rencontre entre différentes cultures peut favoriser le dialogue, elle est parfois aussi source de conflit.

Examinant les mutations qui affectent aujourd'hui les musées, Thomas évoque de manière plus implicite la question des " arts du monde ", car son livre s'intéresse avant tout à la valeur éthique et éducative du musée, en délaissant la question de l'esthétique idéaliste. Il analyse les défis auxquels sont confrontés les musées à partir de la notion de curiosité, qui précède toute considération esthétique ; catégorie heuristique plus souple, non hiérarchique, elle peut être appliquée à tous les musées qu'il évoque, qu'ils soient consacrés à l'art, à l'histoire, à la science ou à l'ethnographie. Son approche illustre donc également le chevauchement ou les relations croisées qui existent entre l'histoire de l'art et d'autres disciplines dont les sources matérielles s'apparentent au patrimoine culturel et transculturel.

Susanne Leeb (2015) aborde la question sous l'angle de l'histoire des idées en remontant au XVIII siècle, à partir d'écrits de philosophes, d'anthropologues et d'historiens de l'art allemand surtout, mais aussi francophones (Herder, Schiller, Semper, Einstein, Riegl, Warburg, Bataille). Selon elle, la catégorie des " arts du monde " est spécifiquement moderne, et son analyse ouvre une perspective diachronique, dévoilant la préhistoire du concept de "globalisme " en histoire de l'art ainsi que sa valeur épistémique, et son enchevêtrement avec le très problématique concept occidental d'universalisme9. L'ouvrage s'intéresse donc à la question du canon et des catégories, selon une approche critique de l'histoire des termes et des concepts ${ }^{10}$, développant un cadre qui pourrait être appliqué à de nombreuses questions transculturelles et cas d'étude, et servir de charnière entre les titres évoqués ici.

\section{Les " arts du monde " en tant que catégorie de mot et d'image}

Leeb montre que la catégorie a été appliquée de diverses manières à des objets issus de différentes cultures pour redéfinir la notion d'art et en faire une forme de différence d'ordre anthropologique, parfois selon une téléologie évolutionniste qui contribue à établir une forme fondamentale d' " altérité " situant dans l'art la distinction essentielle entre l'homme et l'animal (LeEB, 2015 , p. 35-69). Dans cette perspective, chaque culture est examinée en fonction de ses apports au processus créatif, conçu comme une démonstration d'humanité et constituant le degré qualitatif qui le sépare de la "nature " (p. 80). La notion est également utilisée pour situer des groupes et des sociétés par rapport à d'autres oppositions hiérarchiques (civilisé/barbare, par exemple), produisant ainsi les conditions heuristiques qui permettent de faire apparaître certains groupes comme moins humains ou moins avancés que d'autres (p. 84). L'un des objectifs de l'ouvrage est de dévoiler le processus hégémonique à l'œuvre dans la création d'un concept totalisant et dans l'élaboration d'un point de vue singulier. Leeb appelle à résister à cette puissance hégémonique de la notion d' " arts du monde " telle que l'envisagent certains penseurs tout au long du $\mathrm{XX}^{\mathrm{e}}$ siècle en mettant en ouvre la différence et en pratiquant une " histoire enchevêtrée ". Il s'agit notamment de lutter contre l'illusion d'unité créée par la "surface esthétique ${ }^{11}$ " de l'Univers des formes.

À cet égard, l'essai de Didi-Huberman (fig. 1) pourrait être considéré comme un complément, puisqu'il invite le lecteur à redécouvrir le complexe ensemble d'idées sur lequel repose le concept du " musée imaginaire " d'André Malraux et son entreprise éditoriale. On pourrait également lire le portrait qu'il dresse de ce dernier, plus généralement, comme une manière de une manière de montrer la façon dont le caractère essentiellement subversif de l'art peut être domestiqué et institutionnalisé. Ainsi voit-on comment une représentation implicite de la hiérarchie (question abordée par Leeb dans son histoire critique des écrits théoriques sur l'art) exerce parfois un impact dans la société au-delà des milieux universitaires, car elle fait partie de l'inconscient culturel et sous-tend les multiples conventions qui fondent la réflexion sur le musée et le patrimoine. Le désir de l'auteur de se pencher sur l'héritage de Malraux apparaît dès le départ traversé de doutes, et sa mise en œuvre d'une " dialectique historique " s'inscrit dans une critique de l'esthétique transcendantale, dans laquelle il voit une sorte d' " anesthésie " (Didi-Huberman, 2013, p. 16). Il examine les 
paradoxes d'un homme politique dont les écrits sur l'art s'attachent particulièrement à maintenir une distinction claire entre l'esthétique et le politique.

Il s'efforce de mettre en lumière toute la puissance de la métaphore de Malraux afin de la soumettre au réexamen critique. Les écrits de ce dernier, très influents et emblématiques du culte de l'art moderniste, n'ont jamais été une source directe pour les historiens de l'art, et leur ton lyrique comme leur éloquence rhétorique leur confère une qualité anhistorique qui les a toujours rendus suspects aux yeux des spécialistes (p. 78). Afin d'expliquer leur " présence durable, mais peutêtre refoulée ", sans doute renforcée par le rôle important joué par Malraux en tant que premier ministre français de la Culture, Didi-Huberman s'attache à historiciser la généalogie complexe du discours sur l'image et l'art. Il replace le concept de « musée imaginaire " dans le riche environnement intellectuel de l'époque, évoquant divers projets ou musées de papier consacrés à la rencontre des cultures : l'Atlas Mnémosyne d'Aby Warburg (1921-1929), la revue Documents de Georges Bataille (1929-1931), ou encore le film de Chris Marker et Alain Resnais, Les Statues meurent aussi (1953). Prenant comme point de repère le caractère politique, voire iconoclaste, de certains de ces projets, il définit la spécificité du travail de Malraux, de son style et de l'usage qu'il fait de l'image, montrant comment son processus intellectuel, en partie guidé par ces divers projets, finit par s'acheminer vers une perspective unificatrice de l'art. Son penchant pour la vision d'ensemble stylistique et spirituelle et la synthèse globale embrassant toutes les périodes apparaît en définitive s'opposer aux notions de différence radicale que l'on repère dans le travail de ses contemporains ${ }^{12}$.

Walter Grasskamp exprime un autre point de vue sur Malraux. S'il estime que le « musée sans murs » est radicalement différent de l'espace muséal concret, il choisit cependant de faire du musée en tant qu'institution son cadre de référence, et de se concentrer sur les aspects du " musée imaginaire " qui privilégient clairement le visuel au détriment du textuel. La priorité accordée au visuel est particulièrement intéressante si on l'examine en lien avec les réflexions d'un Malraux théoricien de l'art. Grasskamp montre ainsi que c'est ce dernier qui, en dialogue avec Paul Valéry et Walter Benjamin, porte le discours sur la reproductibilité de l'image au-delà du topos de la perte d'aura ${ }^{13}$ (GRASSKAMP, 2014, p. 50-56). L'un des grands mérites du livre, s'agissant notamment de l'utilisation et de la circulation des images, est de rappeler le rôle essentiel joué par deux figures pratiquement oubliées dans l'évolution de la pensée de Malraux. Le premier est le conservateur allemand Alfred Salmony, qui lui expose dès 1923 le concept d'une

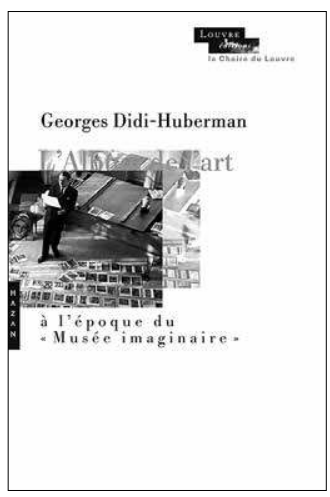

" exposition artistique comparative " conçue à partir d'associations de photographies d'œeuvres d'art. Malraux découvre ainsi comment la photographie peut être utilisée pour inventer une histoire de l'art purement visuelle, et comment d'autres méthodes comparatives plus anciennes (celles imaginées notamment par Henrich Wölfflin et d'autres protagonistes de la Stilgeschichte) pourraient permettre d'envisager un canon artistique d'ampleur plus "globale " (p. 5660). La très innovante Encyclopédie photographique de l'art d'André Vigneau, dont le premier volume est publié à Paris en 1935, est une autre source directe des photographies utilisées par Malraux. Grasskamp, qui a lu attentivement le Musée imaginaire de la sculpture mondiale, montre comment Malraux a systématiquement adapté - en les modifiant - un nombre non négligeable des images et des concepts typographiques de Vigneau à son propre projet qui, reposant sur la métaphore visuelle du " musée " et non sur celle de l' " encyclopédie " de papier, s'est révélé plus séduisante à long terme et a eu une grande fortune ${ }^{14}$ (p. 61-74). Cet épisode suffit à démontrer comment les images, leur circulation et leur encadrement épistémique permettent de redessiner la carte de l'histoire de l'art et de ses canons en expansion ; l'appareil méthodologique était déjà opérationnel et les images disponibles, mais Malraux a fait fusionner les questionnements d'une histoire de l'art largement axée jusque-là sur l'Europe et la Méditerranée avec l'intérêt naissant pour ce que ses contemporains avaient baptisé les " arts du monde ". Le " musée imaginaire " fait partie de ces ouvrages illustrés qui ont sans aucun doute contribué à populariser la notion d' " arts du monde " à partir du milieu du $\mathrm{XX}^{\mathrm{e}}$ siècle (fig. 2 ; GRASSKAMP, 2014, p. 98-130; en part. p. 110).

Mais l'analyse de Grasskamp ne se clôt pas sur ce chapitre consacré à l'histoire de l'art comme
1. Première de couverture de L'album de l'art à l'époque du "Musée imaginaire", de Georges

Didi-Huberman (Paris, 2013), montrant une photographie de Maurice Jarnoux, André Malraux chez lui, publiée dans Paris Match en 1953. 
2. Capture d'écran extraite de la vidéo de Dennis Adams, Malraux's Shoes, 2012, 42 min., Dennis Adams et Paul Colin (réalisation).

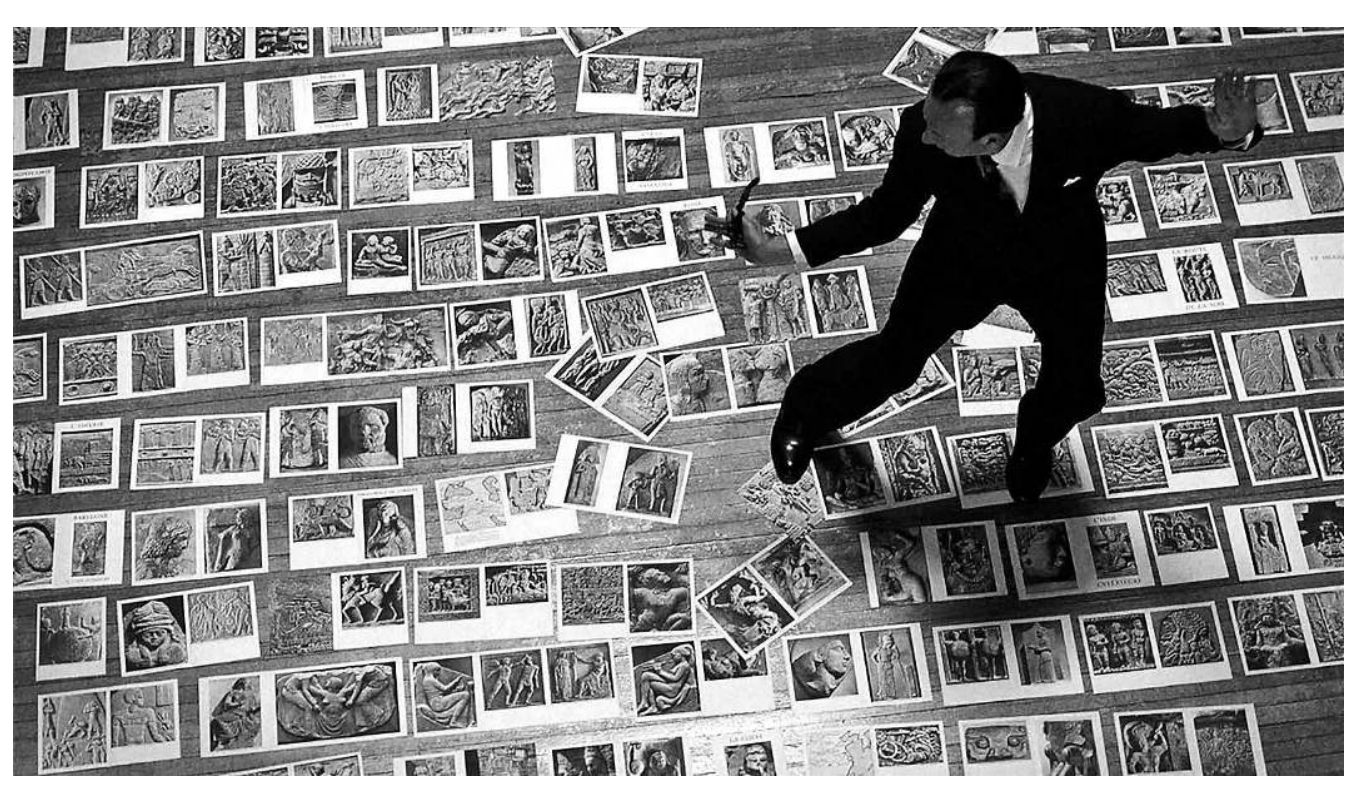

histoire des médias ; celle-ci conduit au « tournant anthropologique " qui a élargi les termes et les concepts de l'histoire de l'art - ainsi que les pratiques et la théorie artistiques - pour leur donner une dimension globale d'un point de vue à la fois diachronique et synchronique (p. 113-130), ouvrant ainsi la voie, d'une certaine manière, à des définitions universalistes et téléologiques de l'altérité et de la différence. C'est sur ce point que Grasskamp rejoint le plus explicitement la perspective historiographique de la critique de l'universalisme offerte par Susanne Leeb (p. 114). Après avoir dressé cet arrière-plan, et évoqué les "primitivismes " hyperboliques du Blaue Reiter à titre de comparaison, Grasskamp distingue deux catégories répondant, pour l'une à la notion d' "enthousiasme ", pour l'autre à une notion dialectique des arts du monde, et qui correspondent à une différentiation qualitative des processus perceptifs apparemment relatifs. Dans le premier cas, on élève " avec enthousiasme " au statut d'œuvre d'art des objets qui, dans leur culture d'origine, ne correspondent en rien à cette catégorie occidentale. Quant à la notion dialectique, elle implique une parfaite conscience des incohérences constitutives de la catégorie ${ }^{15}$. Approfondissant ces différences qualitatives, Grasskamp montre qu'il aurait été impossible, au début du $\mathrm{XX}^{\mathrm{e}}$ siècle, d'aborder sous un angle théorique le concept $\mathrm{d}^{\prime}$ " arts du monde " sans les contributions des anthropologues, mais aussi des artistes, des collectionneurs et des marchands ainsi que des musées qui, autant à travers les questions qu'ils soulèvent que par leur réalité matérielle, ont largement pesé sur les histoires de la perception ultérieure du " musée sans murs ${ }^{16}$.

\section{Exposer, manipuler et interpréter : matérialités de la rencontre interculturelle}

Ces différentes perspectives historiques fournissent un cadre intellectuel permettant de mieux comprendre les crises de la représentation que les musées ethnographiques et les autres musées abritant des collections d' " arts du monde " ont traversé au cours des dernières décennies. La critique conceptuelle proposée par ces différents ouvrages évoque les reproches spécifiques adressés au musée, notamment le fait qu'il dissocie les objets de leur contexte et leur applique des perspectives singulières. Tout cela est d'autant plus problématique que la distribution des collections entre Nord et Sud est extrêmement déséquilibrée, comme l'a souligné Leeb ${ }^{17}$. Didi-Huberman évoque lui aussi la réalité conflictuelle des pratiques coloniales et impériales de la collection, plaçant en exergue de son ouvrage une phrase de Walter Benjamin, souvent citée pour illustrer les ambiguïtés du patrimoine global ${ }^{18}$ : " Il n'est pas de témoignage de culture qui ne soit en même temps un témoignage de barbarie ${ }^{19}$."

L'ouvrage le plus récent, celui de l'anthropologue Nicholas Thomas, directeur du Museum of Archaeology and Anthropology at Cambridge 
University, évoque nombre des grands thèmes de la critique actuelle adressée au musée, examinant les réponses apportées par l'institution. Thomas se présente lui-même comme le représentant d'une génération de spécialistes et de chercheurs qui ont appliqué cette critique au musée. S'adressant à un public de non-spécialistes, il évite de s'attarder sur tel ou tel contexte ou institution, privilégiant l'aperçu général à partir d'un grand nombre d'exemples de musées de types différents, mais essentiellement axés sur l'Europe (fig. 3). L'ouvrage s'attache à mettre en lumière les stratégies mises en œuvre pour assurer la légitimité

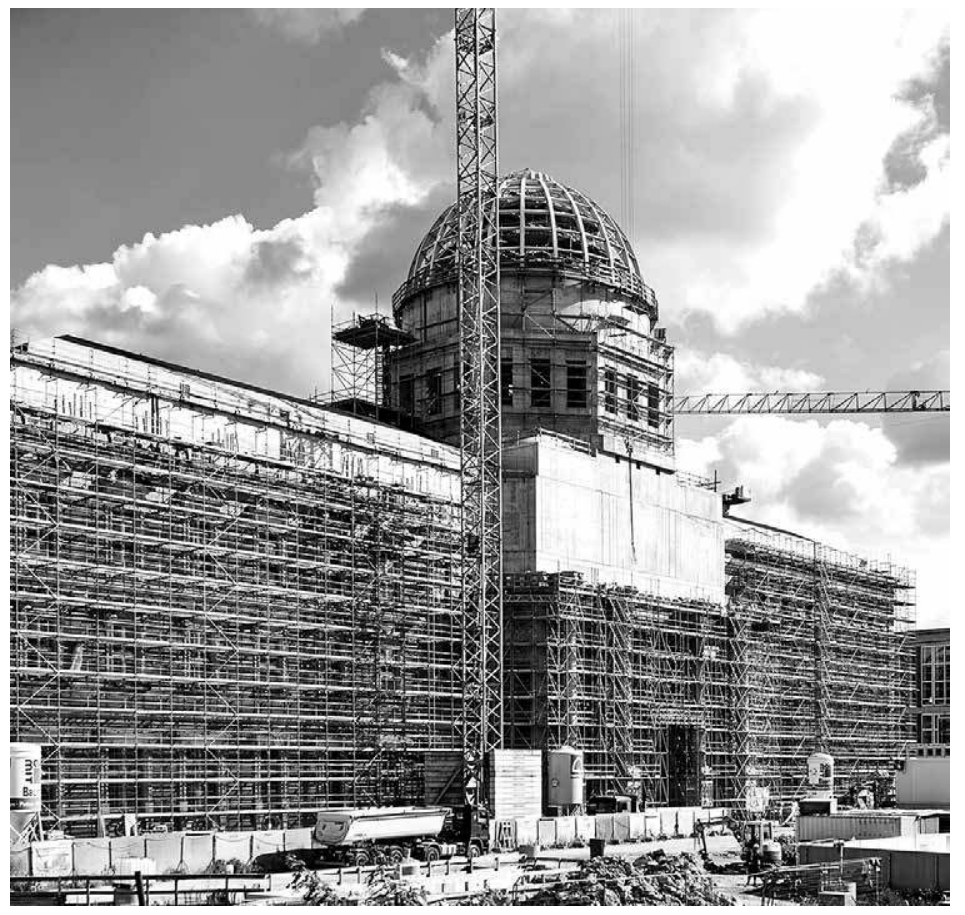

3. Le Humboldt Forum en construction, vue de la façade (porte 3), Berlin, 2017. et la pertinence sociale de

l'institution au XXI ${ }^{\mathrm{e}}$ siècle ; après avoir évoqué "l'ascendant du musée ", il discute les notions de "musée comme méthode " et de " collection comme technologie créative ", notion sans doute d'ordre plus spéculatif. L'un des aspects les plus intéressants de son étude réside dans l'effort de conciliation des deux facettes de l'institution : traditionnellement fondée sur l'objet, elle s'adresse aussi au public, et doit donc expérimenter ${ }^{20}$. La notion de rencontre dans un " musée relationnel " est ainsi un leitmotiv de l'ouvrage.

Thomas parvient à éviter le ton " triomphaliste " (THOMAS, 2016, p. 18) ou utopiste en présentant sa vision du musée d'aujourd'hui et de demain, et reste conscient des difficultés politiques et de la nécessité pour l'institution d'être en prise avec le monde réel, où des valeurs culturelles différentes peuvent être source de tensions. Sa vision générale de la situation des musées comme des espaces consacrés au développement social le conduit à envisager l'institution comme une " méthode et [une] forme d'activité " (p. 100), qui conserve les collections en constant état de flux dans sa structure même, animé par le désir et la curiosité d'engager de nouveaux dialogues, d'observer de nouvelles transgressions des genres et des disciplines (p. 113). Mais cette façon de penser la curiosité pourrait aussi devenir un autre type d'approche universaliste de l'objet, fondée sur la quête de nouvelles connaissances et centres d'intérêt du visiteur ; à cet égard, elle est non moins idéale ${ }^{21}$ que le concept d'appréciation esthétique de Malraux. Dans les deux cas, l'expérience est renforcée par le dispositif, qu'il soit muséal ou photographique, qui isole l'objet afin que celui-ci soit mieux vu ou compris.

Cette curiosité rappelle à certains égards l' " enthousiasme " identifié par Grasskamp comme une composante possible de la perception des " arts du monde ". On pourrait évoquer ici le récent " tournant affectif " emprunté par les sciences sociales, et introduit dans les études visuelles par différents chercheurs, notamment Elizabeth Edwards ${ }^{22}$. Une fois encore, on note que ces approches sont souvent étroitement liées aux questions de représentation et d'interprétation qui se rapportent aux deux catégories du mot et de l'image et qui, dès l'origine, ont forgé la notion de transculturel. Comme l'ont montré de nombreuses études d'histoire de l'art et d'archéologie, la relation triangulaire entre un objet ou une œuvre d'art, sa perception et son interprétation n'est jamais "innocente ", ni dépourvue de préalables, quels que soient les termes du débat et les catégories invoquées. Ce débat, avec ses récentes évolutions (notamment le " tournant affectif ") 
4a. Première de couverture du catalogue de l'exposition Chefs-d'œuvre du delta du Gange, collections des musées du Bengladesh, Vincent Lefèvre, Marie-Françoise Boussac (dir.), cat. exp. (Paris, musée des Arts asiatiques Guimet, 2007-2008), Paris, 2007.

4b. Des manifestants devant le Bangladesh National Museum, contre le déplacement des objets en France pour l'exposition "Chefs-d'œuvre du delta du Gange ».
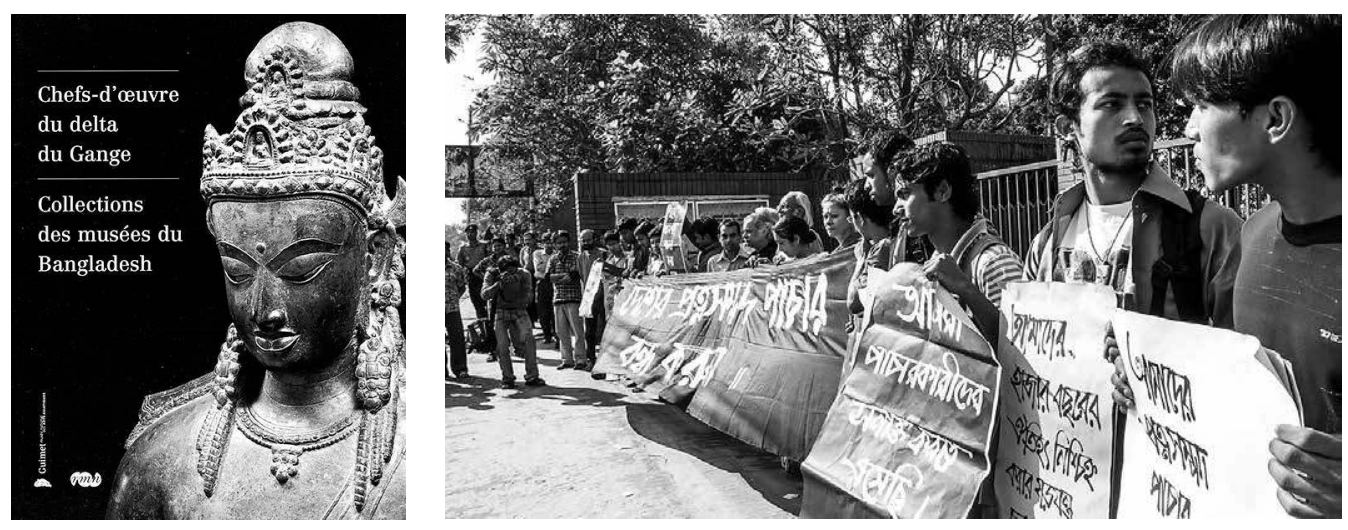

permet d'appréhender de manière plus aiguë les processus de perception transculturelle dans toute leur diversité, et le potentiel épistémique qu'ils recouvrent ${ }^{23}$.

En tant que méthode, la notion de " curiosité » permet, bien entendu, d'ouvrir de multiples perspectives, même si Kavita Singh nous rappelle, à propos des manuscrits sikhs du Government Art Museum and Gallery de Chandigarh (SINGH, 2015, p. 60-70), que, dans le cadre du musée, cette curiosité peut être perçue comme une menace pour des systèmes de pensée en contradiction avec "le cadre séculier de [l']artefact " (p. 58). Citant un exemple qui pourrait, selon elle, servir à illustrer tout un éventail de phénomènes, Singh raconte ainsi que certains dirigeants sikhs locaux, dans un esprit de zèle, ont demandé au musée de traiter ces manuscrits en suivant la pratique religieuse, c'est-à-dire de les dissimuler au regard. La " tradition " peut ainsi être mise en ouvre - et non plus observée - au service d'une religiosité politisée et instrumentalisée " afin de provoquer une perturbation sous prétexte de sensibilité froissée " (p. 67).

À partir d'un autre exemple - la controverse entourant l'organisation de l'exposition "Chefsd'œuvre du delta du Gange ", qui prévoyait le prêt de 188 œuvres d'art par le Bengladesh au musée Guimet en 2007 (fig. 4a et b) -, l'auteure montre la dimension potentiellement négative du trope du musée occidental lorsqu'il est exploité au cœur de querelles politiques locales dans des régions du monde où il représente un modèle importé. Son analyse illustre la « collision entre cultures » dans le musée et dans la définition d'un patrimoine mondial ; elle souligne également les angles morts de l'argumentation contre la restitution des œuvres, tout en défendant par ailleurs l'institution, en tant que lieu d'échange et de partage de connaissances ${ }^{24}$. Si l' $l^{\prime}$ universalisme désuet et bien mal en point " du musée apparaît comme la manifestation d'une pratique culturelle particulière, celui-ci n'en reste pas moins précieux, car il permet de bâtir un imaginaire commun. Les exemples cités par Singh soulignent la contradiction qui existe entre deux perceptions du musée : une institution prédatrice (p. 10-28) ou une " enclave assiégée " (p. 73). Ces deux perspectives opposées ne l'empêchent nullement de prendre position ; dans sa conclusion, elle défend le droit d'accès aux images et aux objets conservés dans les musées, sources de savoir qui permettent de mieux comprendre d'autres cultures.

\section{Des contact zones ${ }^{25}$ aux conflict zones}

Cet aperçu de quelques ouvrages récents a couvert des territoires différents, depuis les traditionnelles conceptualisations bourgeoises et/ou universitaires occidentales, jusqu'aux zones de dispute, voire les " champs de bataille " de la différence culturelle et politique. La genèse de ce que l'on appelle l'histoire de l'art " globale " ou " transculturelle ", dont les répercussions se font sentir dans les milieux universitaires et dans les pratiques culturelles, nous force à disséquer les hiérarchies sociales, les frontières géographiques, les ordres représentationnels, et à remettre en question les histoires linéaires. Comme l'affirme Leeb en évoquant les travaux d'Étienne Balibar, il faut inventer des universalismes multiples ou pluriels (LEEB, 2015, p. 298), afin de passer outre les universalismes formels ou institutionnels, et délaisser les histoires binaires et comparatives pour aller vers l'image polycentrique d'une histoire 
" enchevêtrée ". Une histoire de l'art transculturelle véritablement globale devra s'attacher à contester sans cesse les cadres et les critères traditionnels. Il ne s'agit pas véritablement de révolutionner les catégories, mais plutôt d'étendre le champ discursif de la discipline, car on " ne peut atteindre, mais seulement exiger ${ }^{26}$ " la diversification de l'universalité. L'art, ainsi que ses médias de représentation et ses institutions, deviennent ainsi un lieu de différend permanent, sans cesse en conflit avec ses propres principes et discours ${ }^{27}$.

\section{Ce texte a été traduit par Françoise Jaouën.}

Felicity Bodenstein, postdoctorante, musée du quai Branly - Jacques Chirac felicitybodenstein@gmail.com

Eva-Maria Troelenberg, Kunsthistorisches Institut in Florenz - Max-Planck-Institut troelenberg@khi.fi.it
1. SINGH, 2015, p. 56.

2. Sur la différence entre " art global " et " arts du monde ", voir Hans Belting "From World Art to Globa Art: View on a New Panorama ", dans The Global Contemporary and the Rise of New Art Worlds, Hans Belting, Andrea Buddensieg, Peter Weibel (dir.), cat. exp. (Karlsruhe, ZKM Zentrum für Kunst und Medientechnologie, 2011-2012), Karlsruhe/Cambridge, Mass./ Londres, 2013. Sur les concepts et la terminologie, voir également Monica Juneja, " Kunstgeschichte und kulturelle Differenz. Eine Einleitung ", dans Monica Juneja, Matthias Bruhn, Elke Werner (dir.), Universalität der Kunstgeschichte?, volume thématique de Kritische Berichte, $\mathrm{n}^{\circ} 40 / 2,2012$, p. 6-12; Eadem, "Global Art History and the Burden of Representation ", dans Hans Belting, Jakob Birken, Andrea Buddensieg (dir.), Global Studies. Mapping Contemporary Art and Culture, actes de colloque (Karlsruhe, ZKM Zentrum für Kunst und Medientechnologie, 2009/2010), Ostfildern, 2011, p. 274-297.

3. James Elkins, Is Art History Global?, Londres, 2007 David Summers, Real Spaces: World Art History and the Rise of Western Modernism, New York, 2003; John Onians, Art, Culture and Nature: From Art History to World Art Studies, Londres, 2006. Susanne Leeb dresse un tableau historiographique critique dans "Weltkunstgeschichte und Universalismusbegriffe: 1900/2010 ", dans Juneja, Bruhn, Werner, 2012, cité n. 2, p. 13-25.

4. Voir Viktoria Schmidt-Linsenhoff, Ästhetiken der Differenz. Postkoloniale Perspektiven vom 16. bis 21. Jahrhundert; 15 Fallstudien, Marburg, 2010.

5. Les écrits de Malraux ont suscité un grand intérêt chez les chercheurs et les éditeurs au cours des vingt dernières années. Citons notamment la réédition en deux tomes de ses Écrits sur l'art (2014), et l'ouvrage de Jean-Pierre Zarader, André Malraux. Les Écrits sur l'art, Paris, 2013. Dominique Vaugeois dresse une bibliographie complète de ses œuvres dans Malraux à contretemps. L'art à l'épreuve de l'essai, Paris, 2016.

6. Voir Henri Zerner, "Malraux, ou les Pouvoirs de la reproduction photographique", dans Écrire l'histoire de l'art. Figures d'une discipline, Jeanne Bouniort (trad. fra.), Paris, 1997, p. 145-156.

7. Voir également GRASSKAMP, 2014, et Idem, "The Museum in Print: André Malraux's Musée Imaginaire and André Vigneau's Photographic Encyclopaedia of Art ", dans Melania Savino, Eva-Maria Troelenberg (dir.), Images of the Art Museum. Connecting Gaze and Discourse in the History of Museology (Contact Zones, 3), actes de colloque (Florence, Kunsthistorisches Institut in Florenz - Max-PlanckInstitut, 2013), Berlin/Munich/Boston, 2017, p. 301-316.

\section{SINGH, 2015, p. 9}

9. Sur la question de l'universalisme, voir notamment Leeb, 2012, citée n. 3.

10. Voir également S. Leeb, " Primitivism and Humanist Teleology in Art History around 1900 ", dans Journal of Art Historiography, $\mathrm{n}^{\circ} 12,2015$ (en ligne : https://arthistoriography.wordpress.com/ ; consulté le 8 février 2017).

11. Sur le projet de Malraux, voir LeEB, 2015, p. 292. 
12. Sur Warburg, Bataille et Einstein voir le chapitre " Die Archaik der Moderne oder Die Internalisierung des Primitiven » dans LEEB, 2015, p. 153-185.

13. Toutes les références renvoient à l'édition allemande de l'ouvrage (GRASSKAMP, 2014 ; traduit en anglais par Fiona Elliott sous le titre The Book on the Floor: André Malraux and the Imaginary Museum, Los Angeles, 2016).

14. Ces réflexions sur le livre de W. Grasskamp s'inspirent en partie de l'article d'Eva-Maria Troelenberg, " Images of the Art Museum: Connecting Gaze and Discourse in the History of Museology. An Introduction ", dans Savino, Troelenberg, 2017, citées n. 7, p. 1-27, en particulier p. 24-25; qui fait référence à GRASSKAMP, 2017.

15. "Der enthusiastische übertrug die Nobilitierungsformel auf Bildwerke aus Kulturen, die den europäischen Kunstbegriff weder kannten noch verstanden hätten, und verabschiedete gleichzeitig entscheidende normative Konturen seiner Genese. Der dialektische ist sich dagegen der vielen performativen Ungereimtheiten dieser Universalisierung bewusst. Der Weltkunstgedanke bleibt ja gerade dann eurozentrisch, wenn er die Geschichte seiner willkürlichen und geradezu überstürzten Universalisierung nicht reflektiert und deren Ungereimtheiten überspielt " (GRASSKAMP, 2014, p. 114).

16. Voir en particulier les chapitres "Wirkungsge schichten ", p. 131-146 ; "Imaginäre Museen », p. 147162 ; "Das Museum als Vorstellungsraum ", p. 163-176.

17. "[S]ind die Artefakte der einstmals Anderen doch größtenteils im Besitz des Globalen Nordens und damit nur dessen Publikum zugänglich, aber nicht Personen aus dem Globalen Süden, aus dem sie einstmals abtransportiert worden waren " (LEEB, 2015, p. 297). Leeb a également approfondi le lien entre la théorie et l'idée de musée universel, en évoquant le projet actuel de l'Humboldtforum de Berlin (Leeb, 2012, citée n. 3, p. 19-20).

18. Homi K. Bhabha analyse cette citation dans "On Global Memory. Reflections on Barbaric Transmission ", dans Jaynie Anderson (dir.), Crossing Cultures: Conflict, Migration and Convergence, actes de colloque (Université de Melbourne, $32^{\mathrm{e}}$ Congrès d'histoire de l'art, CIHA, 2008), Victoria, 2009, p. 46-56.

19. Walter Benjamin, "Sur le concept d'histoire " (1940), Euvres, Maurice de Gandillac (trad. fra.), Paris, 2000 , vol. III, p. 433. " Es ist niemals ein Dokument der Kultur, ohne zugleich ein solches der Barbarei zu sein ", Über den Begriff der Geschichte, 1940, VII.

20. Dans Do Museums Still Need Objects? (Philadelphie, 2010), Steven Conn remet en question la fonction de plus en plus conceptualisée du musée en tant qu'espace d'idées, conçu et géré pour servir le public. Lieu destiné à la rencontre entre objets et visiteurs, il fait aujourd'hui naître le soupçon que le musée consacré aux objets et celui axé sur le public répondent à des notions opposées.

21. THOMAS, 2016, p. 61.

22. Voir notamment Elizabeth Edwards, "Anthropology and Photography: A Long History of Knowledge and Affect ", dans Photographies, no 8/3, 2015, p. 235-252.

23. Voir Elizabeth Hallam, Brian V. Street (dir.), Cultural
Encounters: Representing "Otherness", Londres/New York, 2000.

24. SINGH, 2015, p. 9.

25. L'expression de conflict zones dans le titre de l'ouvrage de Kavita Singh fait référence à l'essai " Museums as Contact Zones " publié dans James Clifford, The Predicament of Culture. Twentieth-Century Ethnography, Literature, and Art, Cambridge, Mass, 1988.

26. LEEB, 2015 , p. 299 : «Zudem kann dieser [Universalismus der Vielheit, " universalisme du Multiple " ou " de la multiplicité "], wie Balibar unterstreicht, auch nie erreicht, sondern nur reklamiert werden. "

27. Ibidem : " endloser Anfechtung ". 